Voix et Images

volxetimages

\title{
Poésie est Eisèop : Une entrevue avec Raoul Duguay
}

\section{Richard Giguère}

Volume 1, numéro 2, décembre 1975

\section{Raoul Duguay}

URI : https://id.erudit.org/iderudit/290071ar

DOI : https://doi.org/10.7202/290071ar

Aller au sommaire du numéro

Éditeur(s)

Les Presses de l'Université du Québec

ISSN

0318-9201 (imprimé)

1705-933X (numérique)

Découvrir la revue

Citer ce document

Giguère, R. (1975). Poésie est Eisèop : Une entrevue avec Raoul Duguay. Voix et Images, 1(2), 157-170. https://doi.org/10.7202/290071ar d'utilisation que vous pouvez consulter en ligne.

https://apropos.erudit.org/fr/usagers/politique-dutilisation/ 


\section{Poésie est Eisèop \\ Une entrevue avec Raoul Duguay}

Il me semble que je ne connais vraiment qu'une poésie, la mienne. Cela peut paraître un «trip " de l'Ego. CELA EST un respect des autres écrivains parce que pour les connaître à fond, je n'ai jamais trouvé le temps. II me semble aussi que j'apprends à écrire et que bientôt il vaudra la peine de parler. II y a cette peine à chercher. II y a cette joie à trouver. II y a cette joie à chercher le langage total, celui qui rencontre tous les tons, qui vise l'harmonie de l'envers et de l'endroit simultanément dans un ordre parfait, continu-discontinu comme la loi de la matière et sa conversion dans l'esprit. Le retour à la simplicité du primitif décivilisé, humanisé. Si un poète passe sa vie à écrire, comment pourrait-il ne pas avoir raison. Et quelle différence avec un autre métier? Si mon écriture est ma vie, ce n'est pas JE qui écrit ${ }^{1}$.

\section{GAUVREAU: «CELUI QUI OUVRE LA PORTE »}

R. G. On pourrait commencer par parler des précurseurs de la poésie contemporaine au Québec. Qu'est-ce que tu dois à un poète comme Gauvreau, ou à d'autres qui ont commencé ce que tu continues? Ou est-ce qu'on peut dire cela?

R. D. La relation avec Gauvreau, c'est vraiment une chose à clarifier parce que beaucoup de gens confondent la poésie de Gauvreau et les expériences que je fais. Le travail de Gauvreau était très différent dans le sens que lui c'était un lettriste; moi je ne me considère pas comme un lettriste du tout. Gauvreau était beaucoup plus en relation avec le surréalisme et le dadaïsme, particulièrement le dadaïsme. Personnellement je ne l'ai pas connu beaucoup, je lus ai parlé une fois ou deux et puis la dernière fois que je l'ai vu, je pense que c'était lors de "La nuit de la poésie" (1970); il m'a dit quelque chose qui m'avait bien impressionné : "En tout cas il y aura eu quelqu'un pour t'ouvrir la porte. " Ce qui est juste: il m'a ouvert la porte, mais la grosse différence qu'il y a est la suivante: ce que Gauvreau écrivait, moi je l'improvise à la voix, tandis

1. Ce texte en retrait, et tous les autres, sont des citations d'une lettre de R. Duguay à R. Giguère, datée du 25 févrıer 1974 . 
que ce que j'écris, ce n'est pas improvisé, au contraire. J'écris en tenant compte de la forme de la lettre, de la dimension visuelle, si c'est une droite ou une courbe, le mouvement des lignes, etc. Puis au niveau du son, j'écris en fonction de recherches encore plus poussées. Par exemple la fréquence de telle voyelle en rapport avec telle autre, les palatales, les labiales, les dentales, toutes les données phonétiques, je fouille beaucoup pour découvrir les rapports qu'il y a entre les sons.

R. G. Tu dis que les influences de Gauvreau étaient surtout lettristes, surréalistes et dadaïstes. Est-ce que toi-même tu as été marqué par ces écoles?

R. D. Pas tellement. J'ai aimé cela, j'en ai lu quand j'étais au collège, mais disons que l'influence que ça a pu avoir sur moi s'est manifestée dans le temps de l'Infonie ${ }^{2}$. J'étais alors dans ma période fantastique, dans le fantasmagorique et même dans le mystique, mais mystique-fou, tu sais, fou, "parti» complètement. Et je contrôlais beaucoup moins mes affaires, je travaillais plus dans le spontané.

R. G. À cause du caractère musical de l'Infonie?

R. D. Oui, c'est ça. Toute la différence est là, vraiment. Si je n'avais pas eu la dimension musicale pour faire passer mes affaires, probablement que je serais seulement un écrivain du livre. Mais je suis un écrivain de l'oreille. Moi, c'est beaucoup plus la musique qui m'a appris à faire mes poèmes que la poésie elle-même.

R. G. La musique, oui, mais il faudrait préciser quelle sorte de musique: contemporaine, dodécaphonique, concrète, sérielle. Messiaen, Varèse, les Allemands, Schoenberg, Stockhausen, Berg, Webern, etc.

R. D. Oui j'ai suivi tout cela, j'ai écrit un gros livre là-dessus qui s'appelle Musiques du Kébèk (1971), j'ai rencontré la plupart des musiciens de musique contemporaine du Kébèk. J'ai fait même de la critique de musique contemporaine, de musique pop, du blues et du jazz.

R. G. En effet il y a le blues et le jazz qui t'ont marqué beaucoup au début, un peu comme Paul-Marie Lapointe qui a écrit en se servant des modèles d'improvisation propres au jazz.

R. D. Il y a eu une période où je m'occupais essentiellement de jazz et d'improvisation. Mes deux premiers livres de poèmes, Ruts (1966) et Or le cycle du sang dure donc (1967), ont été écrits à cette période-là; et puis plus tard, du temps de l'Infonie, c'était la musique contemporaine que j'essayais de développer. Maintenant je suis plus tempéré dans mes affaires, plus logique peut-être, et plus circonspect: je définis plus les cadres de mon travail, puis j'approfondis. Probablement que je suis l'un des poètes qui a le plus développé la dimension sonore du

2. Groupe de musiciens et chanteurs dont fit partie R. Duguay de 1967 à 1972. 
poème. En tout cas, au Kébèk, j'en connais peu qui ont fait autant de recherches, mais tu sais, j'ai commencé en 1961, cela fait quatorze ans.

R. G. Gauvreau aussi se servait énormément du matériau sonore du poème, mais tu dirais sans doute d'une façon plus spontanée, moins scientifique.

R. D. Gauvreau vraiment c'est un bonhomme, c'est un taureau, un bloc, un buffle ce gars-là, et d'une importance très grande dans la littérature kébékoise parce qu'il est vraiment l'application du désespoir du poète, du poète qui ne peut pas passer son langage au monde, qui ne peut pas communiquer. Car en réalité, c'est un problème de communication. En somme Gauvreau voulait communiquer mais avec son langage à lui, sans utiliser le langage en place, tu sais, vraiment un "refus global» de prendre les moyens qui sont là. Moi je dis: Il y a eu Refus global, maintenant c'est l'acceptation globale. J'accepte tout, le seul problème, c'en est un d'organisation, parce qu'il ne faut pas que cela prenne toutes les directions à la fois. L'attitude critique et dénonciatrice du manifeste Refus global, dans son temps, était nécessaire. J'ai passé par cette période il y a à peu près dix ans, j'ai fait beaucoup de spectacles politiques qui attaquaient et dénonçaient, j'ai sorti toutes mes "crottes". Maintenant cela ne m'intéresse plus vraiment, c'est la fleur qui va sortir du paquet de fumier qui m'intéresse. Dans certains cas, peut-être que je faisais du défoulement, de l'expression libre si tu veux, mais de plus en plus mon travail s'oriente vers la musique, mes poèmes sont pensés, avec des structures au départ et une organisation précise. On pourrait peut-être parler de poésie concrète.

\section{LA POÉSIE CONCRETE}

La poésie qu'on dit concrète est en fait une forme d'écriture qui ressemble à un DESIGN EN LITOTE, l'épuration du discours jusqu'à le laisser percevoir comme une pulsation, un trait, un rythme, une permutation de chiffres et lettres un dégagement d'espace un mouvement de main pour l'œil mais surtout: une répétition des mots des syllabes des lettres des formes à répétition ce qui donne en mon cas la yantra-mandala

mais toujours: UN JEU dehors

$$
\text { une rotation }
$$

jusqu'à la fin: CEIL QUI REGARDE LE REGARD DU DEDANS

une joie de créer

comme si les mots les syllabes les lettres les SIGNES

devaient inventer un ordre nouveau dans le désordre ap-

parent de la syntaxe et du lexique

comme une grammaire logique en sa derasson

le pouvoir de la parole qui se dépossède de lui-même

dans un dessin plein de desseins de seins d'amour

une folie permise pour arrêter le langage de crier

une danse soudaine dans une architecture de sons 
cela est très passionnant pour un écrivain

de savoir quand, ne parlant pas, il parle...

R. G. C'était la question que je voulais te poser: si tu te sers du matériau du langage, si tu conçois le langage comme un matériau sonore, visuel, plastique (comme la pierre ou le bois pour un sculpteur), alors tu fais de la poésie concrète... mais tu ne voudrais pas qu'on attache cette étiquette à ta poésie?

R. D. Ce que je ne veux pas, c'est qu'on me colle une étiquette alors qu'au fond ma recherche est globale. En même temps que j'écris des poèmes très structurés, très travaillés, d'un autre côté j'essaie de faire de petits poèmes très simples. J'aime cela organiser la matière, j'essaie d'exercer ce que je considère être la fonction du poète, qui est de faire, de créer (comme l'indique la racine grecque "poïen"). L'acte de la création en soi, c'est la poésie. Par-dessus tous les autres arts, c'est l'acte de la parole qui m'intéresse. Je me dis qu'il y a bien des langues qui se parlent sur la terre et qu'il y a donc beaucoup de possibilités offertes. Actuellement je suis en train d'écrire en plusieurs langues pour justement dépasser le problème de la langue. Ce qui m'agace vraiment, même si je suis avant tout kébékois, c'est de me faire limiter à ce point de vue.

R. G. Tu veux dire que tu ne veux pas te limiter à certains sons seulement, tu veux déboucher sur d'autres sons, la langue allemande, l'italien, ce sont d'autres sons?

R. D. Oui parce que c'est la vibration qui m'intéresse comme la source même du poème, c'est le pouvoir énergétique, le potentiel énergétique qui est contenu dans certains types de sons. Ce qu'on appelle poésie concrète finalement, c'est une sorte de désarticulation, de déshabillage de la lettre, de la syllabe, du mot, de la syntaxe.

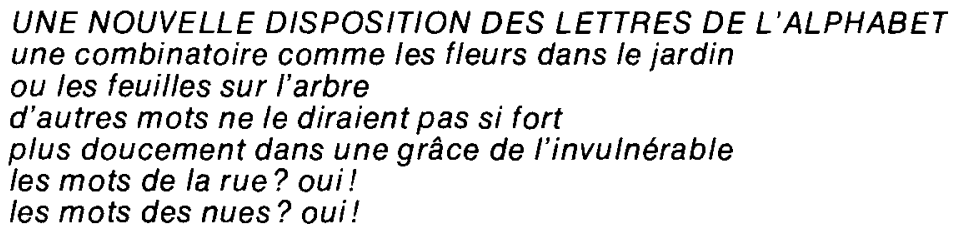

R. G. Tu ne limites donc pas ta poésie en y apposant le mot "concrète", parce que les deux caractéristiques dont tu viens de parler, d'un côté jouer avec les sons, tous les sons possibles, d'un autre côté que cela ne soit pas limitatif mais demeure ouvert sur d'autres horizons, tout cela fait partie de la poésie concrète. La poésie concrète existe actuellement parce qu'elle n'a pas été définie pour être limitative, pas encore du moins : elle s'occupe de toutes les dimensions du poème.

R. D. Je suis d'accord avec toi là-dessus, mais d'un autre côté, cela dépend de quel genre de poème tu parles, quel exemple tu prends. Je ne m'oppose pas à ce qu'on me place dans toutes sortes de catégories de poè- 
tes, pourvu qu'on garde les avenues, toutes les avenues ouvertes, voilà l'important. Je reviens sur l'histoire de Gauvreau pour donner un exemple. Le désavantage que Gauvreau a pu avoir, c'est de dire ses textes d'une manière tellement sérieuse, crispée, se repliant à ce point sur luimême, que le monde n'a pas voulu "embarquer". Si tu l'avais vu derrière la scène quand il était pour lire un poème, il lisait ses textes et il tremblait comme une feuille: "Gastribig aboulcrouc nouf geûleurr naumanamana manamouèr. " Il les lisait, puis il voulait être "touttt " tu comprends, il voulait atteindre le point de perfection dans la sonorité et le rythme, parce que Gauvreau, c'est un magicien, un magicien du son. Je veux dire, quand tu écoutes ces sons!!!

R. G. Mais ces sons-là, cette espèce... on pourrait dire d'agressivité, ne vient-elle pas du fait qu'il a été refusé tellement longtemps comme poète, qu'il criait dans le désert? Le groupe des Automatistes, à la fin des années 40 , été réduit au silence. Borduas a dû s'exiler, c'était difficile de se rendre aussi loin dans la contestation, dans le temps. Toi tu arrives après et tu peux être plus « $\mathrm{CoOl}$ » dans tes spectacles; comme l'a dit Gauvreau, la porte est ouverte.

R. D. Venant après Gauvreau, ce que je fais, on l'accepte plus facilement. On me trouve farfelu, bizarre ou fantaisiste mais je n'écris pratiquement pas de poésie fantaisiste, au contraire. Je suis peut-être fantaisiste dans la manière de présenter mes spectacles. Je suis un des seuls poètes qui chantent, en plus avec un groupe de musiciens. Alors je réussi à faire passer les poèmes; tout le travail qu'a accompli Gauvreau, moi je vais être capable de le pousser beaucoup plus loin et le public va marcher. Au bout de la ligne, cela va me donner une œuvre musicale qui est basée sur un poème; c'est un poème qui est devenu musical.

R. G. Beaucoup de gens qui assistent à tes spectacles et qui ne te lisent pas vraiment pensent que tu écris au fil de la plume. Si tu nous parlais de la façon dont tu composes un texte.

R. D. Je travaille à mes textes avec des séries rythmiques régulières, calculées, organisées; et puis si je laisse de la place à l'irrégularité, eh bien c'est vraiment de l'improvisation, mais tout le reste je le contrôle. Le reste c'est écrit, c'est pensé, il n'y a pas de gratuité. Je n'écris pas au fil de la plume. Si je le fais je me ramasse avec des tonnes de papier et je me dis: j'aurais bien pu prendre mon temps et en faire seulement deux pages, je fais très attention à cela. J'écris quand même un premier jet, disons que j'ai l'idée de faire un poème, j'écris, je laisse aller tel quel et puis, à un moment donné, il vient une phrase ou un son, un mot qui est comme le noyau du texte. Tout le reste c'est comme la chair, tu as le cœur et là tu amènes le sang, tu enveloppes le tout pour que ça se tienne. Enfin il y a le travail, le travail de tout organiser, réécrire, on "tombe" dans le travail. 
La poésie que je produs est eisèop lorsque je viens pour la définir je ne peux plus la poésie est expression de l'infini

R. G. Voici quelques exemples de "concrete poetry" canadienne-anglaise. (Je montre à Duguay The Cosmic Chef, l'anthologie canadienne de poésie concrète publiée par B. P. Nichol en $1970^{3}$.)

R. D. Dans le dessin que je vois là, le poète a fait un cercle et il a mis des mots dedans, où est-ce qu'il est le poème? Regarde, il écrit tunnel comme ceci: TחกกEL.

R. G. C'est un U à l'envers pour imiter la forme d'un tunnel, mais tu lis bien tunnel d'un seul coup d'œil, tu n'as aucune difficulté à le lire?

R. D. Non, aucune difficulté, tu vois la forme qui est là, l'endroit et l'envers; au fond, on a des illusions d'optique; automatiquement, quand tu vois cela, tu dis non, ce n'est pas écrit normalement, mais on sait que c'est tunnel, donc c'est là qu'il est, le poème. Cela me rappelle, il y a un poète, un Canadien anglais qui m'a écrit pour m'envoyer une affaire extraordinaire qui s'intitule: $A L L O$. II m'expédie ce livre-objet chez moi, me dit que personne n'apprécie ce qu'il fait mais que moi je devrais comprendre. C'est tellement simple que... et puis c'est de la poésie.

R. G. Quand tu vois l'objet c'est tellement simple que tu saisis tout de suite, et pourtant...

R. D. Oui, oui, c'est simple mais lui est allé au bout de son idée, voilà l'extraordinaire, la poésie! II a fait un «a" à la main, en gros, calligraphié, et à la suite trois "l » sur la première page. Tu tournes la page, de l'autre côté, ce sont des «IIIIIII" alignés, tout pris l'un dans l'autre, mais seulement des "l», en tout, je ne sais pas, peut-être quatre-vingts, page après page et la dernière finit ainsi: "llllo". Il est fou le type qui m'a envoyé ça, mais il est parfait “en tabarouette" parce que le poème, c'est d'avoir osé écrire dans tout un livre «allo», c'est un livre complet. Alpha sur la première page du livre et oméga sur la dernière. Alpha et oméga, si tu réfléchis à ce que cela veut dire, c'est tout un programme poétique: voilà le poème, eh bien moi, je l'ai trouvé génial ce poète. Il a aussi inclus un autre objet dans son envoi, il a fait un livre sur le SaintLaurent, alors tu ouvres le livre: partout des vagues, oui, le papier est découpé.

R. G. Tout en relief?

R. D. Le papier est découpé et tu peux faire bouger les vagues. Tu vois, ça c'est de la poésie concrète: il n'y a pas un mot mais c'est de la poésie, parce qu'il a pris l'image, il l'a transcrite dans la matière et puis il a dit ce qu'un poème écrit avec des lettres ne peut pas dire.

3. Toronto, Oberon Press. 
R. G. En effet il faudrait des milliers de mots pour dire ce qui se passe dans ce poème-objet sur le Saint-Laurent, et encore.

R. D. Le charme serait perdu.

R. G. Platement, ce serait analytique.

R. D. Il y en a qui font vraiment des choses qui sont inspirées, il n'y en a pas beaucoup, mais it y en a. Même parmi les poètes lettristes, certains réalisent des poèmes extraordinaires, des dessins...

R. G. Ils se servent de la graphie et de la calligraphie.

R. D. Exactement.

R. G. Tu trouves que d'une façon générale, au Canada anglais, il y a plus d'argent pour produire et imprimer ces livres et objets? Est-ce que les exemples que tu viens de fournir seraient difficiles à publier ici ? Quel éditeur s'en chargerait?

R. D. Imagine, tu trouves un imprimeur et tu lui dis, voilà, je voudrais que toutes mes pages soient découpées comme ceci, en vagues. L'imprimeur répond par un "ouais, écoute, il faut se prendre de bonne heure, il faut faire des plaques, des moules, cela coûte tant". Le poète est bien obligé de dire qu'il n'a pas d'argent, c'est loin d'être facile.

R. G. Parlant d'imprimeur et d'édition, qui est-ce qui t'a encouragé à écrire et à publier, en début de carrière?

R. D. Miron, mais pas à publier, pas au départ. II me faisait lire, parce que la première des choses, il faut apprendre à lire, on n'écrit pas sans savoir lire, autrement cela ne tient pas debout. On a oublié ce principe d'aitleurs, on a oublié la lecture, et pourtant elle est corrélative, immédiatement corrélative à l'écriture. Écriture et lecture vont de pair, et de plus en plus pour moi écriture et graphie, calligraphie vont ensemble.

POÉSIE MARGINALE, veut dire LECTURE MARGINALE autre chose, une autre approche de la lecture des signes, celle que l'on peut inventer soi-même selon les lois; pour moi la loi confirme la règle dans sa liberté totale mais il y a un choix: futur, passé ou présent?

R. G. Explique un peu, donne des exemples.

R. D. Là, tout à l'heure, quand j'écrivais le «l». Je vois le geste poétique physique, regarde et écoute le "beat" que ça donne: "lelellel", seulement le geste de reproduire cette forme-là. Pour pouvoir écrire la lettre, il faut mettre tant de temps: faire l'angle, l'ellipse, revenir, etc. Le « $\boldsymbol{Q}$ », c'est tout un «beat» différent, tu peux l'écrire lentement ou rapidement, mais il y a un «beat» pour produire la lettre. Et puis le « $\Omega$ », tout saccadé, et il y a d'autres lettres, tu ne peux pas les faire toutes à la même vitesse, c'est une loi qui est dans la main. 
R. G. Calligraphie et loi de la main, quelle est la relation? Qu'est-ce qui t'intéresse dans la formation des lettres de l'alphabet?

R. D. La main qui à un moment donné a tracé un « $A$ »... un enfant qui trace un " $A$ ", il ne sait pas que sa main a fait ce dessin; il ne sait pas non plus que le “ $A$ » est la première lettre de l'alphabet, qu'il représente l'espace, qu'il correspond à l'échelle et au compas. Prends un compas et puis regarde la forme, le « $A$ » mesure l'espace. Le " $O$ " lui est le résultat de l'espace, c'est le temps qu'il a fallu pour pouvoir faire l'espace. Donc, dans les textes anciens, on dit: “ $A$ » représente l'espace et “ $O$ »le temps. C'est vraiment fantastique! Moi cela m'intéresse de savoir ce qui est arrivé, d'où il vient le «i», comment il se fait que l'écriture de cette lettre produit deux mouvements, deux sons, comme ceci: “ $i$ ", ting-tong. Et on pourrait continuer ainsi avec chacune des lettres, consonnes et voyelles.

\section{LA POÉSIE ET LE POLITIQUE}

R. G. Pour passer à un tout autre ordre d'idées, j'aimerais aborder le sujet poésie et politique. C'est un thème qui a marqué profondément les années 60 en poésie québécoise. Ta poésie a été influencée par le politique au début, tu as tenu une chronique régulière dans la revue Parti pris en 1965-1966.

R.D. Un jour, je me suis assis et je me suis demandé quelle était ma fonction spécifique à moi, quelle était ma place, et c'est là que j'ai découvert que j'étais poète. J'ai découvert aussi que c'était sérieux et important au point de vue social, politique, culturel, etc. Je suis donc poète, qu'est-ce que je vais faire? J'en ai conclu que tout ce que j'avais à faire, c'était de la poésie, la plus belle possible, et de la rendre la plus comestible possible sans baisser mes culottes. Le geste le plus politique que je peux poser actuellement c'est d'écrire le plus beau des poèmes, en tenant compte de toute l'existence humaine.

R. G. Cela ne veut sans doute pas dire qu'il n'y aura plus aucune référence politique dans tes poèmes, puisque, comme tu le dis, si tu tiens compte de toutes les dimensions de l'humain, il y aura toujours un aspect sociopolitique. Mais les textes à haute densité politique seront moins nombreux.

R. D. Attention! j'ai un livre que je veux faire paraître sur la politique; c'est un livre complet que peu de gens connaissent. Mais un jour je veux le publier parce que je trouve que c'est une question sérieuse.

R. G. Excuse-moi de m'attarder sur cette question, mais un des reproches que des jeunes poètes comme François Charron et le groupe de Stratégie adressent à d'autres poètes, ceux de la Barre du jour entre autres, c'est que leurs textes ne sont pas assez politisés. C'est étrange parce que les préoccupations politiques qui avaient quelque peu disparu de la 
poésie à la fin des années 60 et ces dernières années semblent revenir maintenant en force. Qu'est-ce que tu en penses?

R. D. Ce qu'on oublie, c'est que j'ai passé par là : j'ai écrit des textes politiques, j'ai enseigné Marx et j'ai étudié la philosophie politique. Ce qui m'intéresse, c'est beaucoup plus les fondements. On voudrait que je m'intéresse, aux détails, mais pour moi les détails, c'est comme le processus du miroir, que je n'ai jamais aimé dans le domaine des arts. Certains poètes choisissent les éléments qu'ils n'aiment pas dans la société, ils les transcrivent, puis ils disent: voici, j'ai fait un poème politique. Pour moi ce n'est pas vrai, ce n'est pas de la politique, c'est du potin, il y a un bon paquet de poètes potineurs. C'est pour cette raison que j'ai de la difficulté à lire de la poésie souvent, c'est du potinage; il n'y a pas de vision, premièrement; deuxièmement il n'y a pas d'épuration dans les formes et enfin, il y en a beaucoup qui n'ont vraiment rien à dire.

R. G. Alors, quelle est la poésie et quels sont les poètes que tu lis, en qui tu crois?

R. D. Je crois surtout aux poètes qui durent, actuellement je crois à un poète qui a publié il $y$ a dix ans et qui travaille encore. Mais certains font paraître un ou deux livres et aussitôt ils veulent se promener avec l'étiquette «poète». Dans mon cas je considère que dans quelques années je serai prêt à écrire la poésie que je veux écrire; pourtant j'ai fait cinq ou six livres mais je ne crois pas que je suis encore vraiment dans mon élément. C'est comme si je me préparais: je suis encore un néophyte en poésie. Alors forcément je juge les autres très sévèrement parce que moi-même je me juge plus sévèrement encore. C'est la poésie qui gouverne, ce n'est pas moi, parce que la poésie est plus grande que moi. C'est comme la musique, je veux dire un musicien, où est-ce qu'il prend sa musique? II y a la musique qui est là, quelque part, il y a les ondes qui circulent; le bonhomme, il se branche ou il ne se branche pas.

R. G. Pour conclure sur le sujet de la poésie et de la politique, il n'y a donc rien qui ressemble à un programme politique pour les années à venir.

R. D. Bon, j'ai fait des textes sur l'identité du Québec qui m'apparaissaient importants, et maintenant je définis mon programme politique d'une manière très simple: c'est d'écrire le mot KÉBĖK, qui est l'écriture originelle de Québec. Dans l'église Notre-Dame-de-la-Victoire j'ai vu une inscription du XVIIe ou Xv\|le siècle qui m'a étonné. C'était marqué: "Deus Providere Kebeka Liberata", qui se traduit à peu près par: "Que Dieu amène la libération du Kébèk". Pourquoi est-ce qu'ils écrivaient alors "Kebeka"? C'était écrit en latin, mais le latin ne change rien à l'affaire. Je l'ai vu ensuite ce mot dans une vieille carte d'Indiens. C'est curieux, me suis-je dit, qu'est-ce que cela veut dire? Kébèk veut dire "Passage", pour moi KÉBĖK c'est poésie tout court, c'est déjà le sens fondamental de la poésie qui est donné dans ce mot-là, c'est tout inclus dans «passage". 
Le poème est à la fois concret et abstrait, à la fois et en même temps, objet et sujet. Un potentiel énergétique. Une bombe de paix dans les ténèbres intérieures. Un éclatement vers la transparence: le réservoir du SILENCE. Le poème essentiel ne peut être que SILENCE. Alors l'écriture doit disparaître sans qu'on le sache et sans résistance plutôt comme un appel de l'innombrable cherchant à manifester l'UN.

Le poème est physique et métaphysique à son gré pénètre les arcanes du rêve et trace le chemin de la réalité mais comment le dire aussi clairement que le soleil brille

\section{LES ARTS DU SILENCE}

R. G. Tu me disais tout à l'heure que tu vas finir par faire de la peinture. Tu as travaillé avec un peintre dernièrement et tu as découvert une nouvelle dimension dans l'art pictural.

R. D. Quand je dis que je vais finir par la peinture, ce que je veux signifier c'est que je m'en vais vers les arts du silence. Moi qui pendant la plus grande partie de ma vie aurai parlé comme personne, et dont tout le contenu est de dire que j'al hâte de tomber dans le silence, voilà qui peut sembler contradictoire.

R. G. Cela peut paraître contradictoire en effet, mais peut-être que parler de cette expérience assez unique que tu as vécue avec le peintre québécois Edmund Alleyn pourrait nous aider à comprendre. Tu as écrit des poèmes qui accompagnent les phötographies de son catalogue d'exposition Une belle fin de journée (1974). D'abord comment se fait-il qu'il t'ait choisi?

R. D. Alleyn est venu me voir parce qu'il savait que je m'intéressais à toulmonde", et il voulait qu'on travaille ensemble. Comme de raison, il avait déjà réalisé une bonne partie de son projet. Pendant deux étés il a photographié des touristes à l'Expo de Montréal, puis il a reproduit un certain nombre d'hommes, de femmes et d'enfants, grandeur nature, sur des panneaux de plexiglass transparent. La reproduction est absolument réaliste, fidèle, minutieuse dans les plus petits détails. Derrière les panneaux il y a six grands paysages avec des soleils, de vrais soleils de carte postale. En somme un travail de fou, qui lui a pris plus de deux ans. À sa demande donc je suis allé voir ses panneaux, je me suis assis au milieu de ses personnages: un concierge, une bonne de maison, une secrétaire, un professeur, une danseuse de cabaret, une serveuse, différents types sociaux. Pour pouvoir faire un texte là-dessus, je me suis dit que j'allais tout écrire, calligraphié au phonème, au son, le plus simple possible.

R. G. Et qu'est-ce que cela a donné?

R. D. Le résultat est que les gens se lisent et ne se reconnaissent pas. J'ai pourtant repris la façon exacte dont les gens parlent au Kébèk et si 
tu lis tel quel, tout haut, il n'y a pas de distorsion. C'est le plus loin que je me suis rendu dans cette veine... L'éditeur du catalogue, le ministère des Affaires culturelles, a trouvé que j'étais allé trop loin: on m'a reproché de briser le langage. Mais Alleyn m'avait bien dit qu'il désirait rejoindre le monde ordinaire. Ce qui est aberrant, c'est que le monde ordinaire ne comprend rien à ces textes, les gens s'imaginent qu'il s'agit d'une autre langue.

Cette période représente, dans mon cas, une forme de poésie parmi tant d'autres et ma poésie doit faire sa vie toute seule; une fois qu'elle est là, ce n'est plus mon affaire, je continue. Par rapport à la poésie traditionnelle je situe ma poésie en 1974 et en avançant "toultemps".

\section{LA LANGUE DU POÈTE}

R. G. C'est sans doute que les gens ne sont pas habitués à lire au son, surtout en ce qui concerne la langue française.

R. D. En effet, on écrit d'une manière et on parle d'une autre, ce qui n'a pas de sens. Le débat joual-français international est assez complexe, parce qu'on ne réussit pas à s'entendre sur une transcription des phonèmes. Prenons l'exemple de quelques mots comme: "de l'autre bord", ce qui donne au son: "de lôt bâr". Le "au " et le "re» de "autre " disparaissent, le «o» de "bord" devient «â" et on ne prononce pas le " $d$ ". Il y a donc des tas d'illogismes entre langue parlée et langue écrite. Par contre, par rapport à la tradition, je tiens à conserver un français qui sera lu par tout le monde, y compris des Français de France. Je n'écris pas seulement pour les Kébékois. Mais le Kébékois, son propre langage, il ne le reconnaît pas.

R. G. Mais on lui a donné un tel complexe d'infériorité linguistique, on lui a dit tellement longtemps qu'il parlait «mal», qu'il ne savait pas articuler, et en plus qu'il ne lisait pas. Depuis qu'il a commencé à croire qu'il ne parlait pas le "bon français" et a essayé d' "améliorer" sa langue écrite et parlée, on ne peut maintenant lui demander de tout oublier et de se mettre à écrire comme il parlait ou comme il parle encore.

R. D. C'est pour faire prendre conscience de cela que j'ai écrit ces textes. C'est une forme d'écriture, car ceux qui ont utilisé le joual, le québécois populaire, depuis dix ou quinze ans n'ont pas poussé leur expérience au bout à mon avis. La transcription phonétique du joual n'a pas connu ses limites, loin de là.

R. G. Tu trouves que le joual, tel qu'employé en littérature et au théâtre, est trop facile?

R. D. À partir du joual, je trouve qu'un auteur comme Michel Tremblay n'a pas refait un langage, il n'a pas été jusqu'à l'extrême de cette logique du joual. 
R. G. Même s'il s'agit de théâtre et que l'auteur affirme qu'il écrit comme ses personnages parlent, tu voudrais qu'il aille plus loin, que ça devienne le langage de Tremblay et non seulement la reproduction de la langue parlée par les gens.

R.D. Je ne suis pas tellement d'accord avec le processus du miroir: par exemple la bonne femme dans les Belles-Sœurs qui ramasse ses timbres. Les gens qui assistent à la pièce pensent à leur vie à eux et aiment se revoir. Aussitôt que tu veux les amener à élargir leur champ de vision, ils reviennent à leur sécurisante «petite vie". On ne se reconnaît pas làdedans, qu'ils vont te dire. Ils en viennent à aimer leurs limites, alors que tout est dans le dépassement de soi, les extrêmes!

R. G. Le processus du miroir est donc jusqu'à un certain point une solution de facilité?

R. D. Je suis contre, philosophiquement contre. Parce que la fonction du créateur, au théâtre ou ailleurs, c'est de transformer la réalité. Le reste, les «média» le font tous les jours, à satiété. Je ne suis pas contre le fait de prendre les données de gens ordinaires, mais qu'on les retransmette d'une autre manière, qui soit le fait d'une pensée créatrice. La pensée du créateur dépasse le niveau «processus du miroir». Et cela entraîne une certaine responsabilité au niveau de la conscience.

D'où ça vient? D'en haut d'en bas de gauche de droite d'en avant d'en arrière ça vient de partout à la fois chacun son tour qu'on le dirige ou pas le soleil finit toujours par se lever ou se coucher ou se coucher et se lever ça vient des yeux des oreilles de la chair de l'esprit des choses des étoiles des images des minéraux des végétaux des animaux ça vient des hommes et de tous leurs règnes sur l'abîme ça vient d'une bulle bleue diaphane et sans royaume ça vient des tripes

\section{UNE POÉSIE UBIQUISTE}

R. G. Un dernier sujet: tes projets pour l'avenir. Tu as déjà fait paraître cinq ou six livres, qu'est-ce que tu envisages de publier prochainement? Qu'est-ce que tu as sur ta table de travail? Bien sûr, il y a ce microsillon.

R. D. Ce disque est un livre pour l'oreille, c'est un autre médium. Comme pour la peinture dont j'ai parlé tout à l'heure, c'est un nouveau monde à explorer. Avec la peinture la poésie de Duguay devient «visible». Cet été je vais faire une sculpture à Matane: c'est comme une caverne dont les parois seront couvertes de poèmes. Les gens qui visiteront ne viendront pas pour lire les poèmes de Duguay, ceux des livres. Ils viendront habiter un lieu et liront les textes sur les murs. Je veux me servir du plus grand nombre de moyens de diffusion possible. Le livre est un moyen, mais ce n'est pas le seul. Aujourd'hui il y a de plus en plus de moyens de faire le même poème. 
R. G. Pour toi, le microsillon est un moyen de faire passer le poème?

R. D. Oui. Mais dans les livres je ne fais aucune concession. Je ne me dis pas: “Je vais écrire pour tel genre de personnes." De plus en plus toutefois, je veux devenir simple, je veux que mes textes deviennent facilement accessibles. Je m'apprête à publier $O$ ou l'Invisible enfant, un livre de 99 pages, sorte de grand mantra, début de cosmogonie. Le pendant de ce livre sera l'Enfant terre, qui, au point de vue de la recherche, est un des textes les plus poussés que j'ai écrits. II n'y a que la série "L'apocalypse" dans Lapokalipsô (1971) que j'ai travaillée autant.

R. G. Côté théâtre, je sais que tu as présenté une pièce, il y a environ deux ans, qui avait connu beaucoup de succès.

R. D. J'ai quelques pièces que je veux publier. D'abord Allo toulmonde que je suis en train de monter actuellement et que je jouerai du 20 mars au 10 avril à Montréal. Pendant cette période de temps il y aura un maximum de deux mille deux cents personnes qui assisteront au spectacle, qui entendront mes poèmes. If $y$ a des gens qui aiment le théâtre, tu comprends; si tu veux les rejoindre, if faut que tu ailles à eux. D'autres écoutent de la musique: alors je fais des microsillons. Je vais là où sont les gens, tous les gens; l'idéal, c'est d'être partout: une poésie ubiquiste. D'un côté, je suis chanceux parce que j'ai des possibilités techniques et un talent pour rejoindre les gens, mais d'un autre côté je suis en apprentissage perpétuel.

R. G. Tu joues toujours avec de nouveaux media qui te demandent un recyclage constant.

R. D. Je suis toujours en train d'étudier pour savoir comment m'y prendre pour reproduire ceci ou cela.

R. G. Comment as-tu aimé ton expérience au cinéma avec $O$ ou l'Invisible enfant? Est-ce que tu as l'intention de reprendre cette direction un jour?

R. D. J'aime ce poème que j'ai fait au cinéma. Si j'en entreprends un autre, c'est sûr que je le ferai d'une façon différente. Avec l'expérience acquise, je pourrais aller plus loin. J'ai l'intention de revenir au cinéma plus tard. Actuellement je concentre mes efforts du côté musical. Depuis un an et demi j'ai écrit quarante chansons à peu près. J'ai de Ja matière pour enregistrer cinq «longs-jeux». Toutes ces dimensions, poésie, peinture, musique, cinéma, sculpture et décors, éclairage pour ma pièce de théâtre, toutes ces dimensions-là sont pour moi le même poème qui circule. Mais essentiellement je suis un écrivain du livre.

R. G. Tu as parlé de plusieurs livres à publier. À part les deux nommiés, quels sont les autres?

R. D. J'ai commencé avec Lyson un livre sur le temps, c'est elle qui fait les dessins. De plus en plus je veux utiliser le côté visuel, les images, le montage, pour que cela soit beau. Le livre est basé sur la structure du 
temps. Puis il y a un autre livre que je projette: un alphabet phonétique rythmique, un genre de méthode d'apprentissage de l'alphabet, que je veux proposer au ministère de l'Éducation. Mais une méthode destinée à l'oreille, un alphabet chanté. Enfin j'aimerais écrire un roman, ce que je n'ai jamais fait. J'irais l'écrire en Abitibi, lieu de mon enfance. Un romancier raconte toujours une histoire, son histoire jusqu'à un certain point. Je raconterais donc la mienne.

Bon, et tout cela s'en va vers le silence.

Ca a commencé dans le ventre de ma mère la terre etc

Ca a commencé par un sapin illuminé de soleils dans le salon chez nous quand j'avais 13 ans, peut-être à cinq ans quand je me suis coupé dans la main gauche avec un couvercle de boîte de café Chase \& Sanborn C'A TOUJOURS ÉTÉ 